\title{
Protective Action of Nanodiamonds Against Influence of Ionizing Radiation in Rats
}

\section{Batyuk $\mathrm{L}^{\mathbf{1}}$ and Kizilova $\mathbf{N}^{2,3 *}$}

${ }^{1}$ Kharkov National MedicalUniversity, Ukraine

${ }^{2}$ Warsaw University of Technology, Poland

${ }^{3}$ V.N. Karazin Kharkov National University, Ukraine

*Corresponding Author: Kizilova N, Warsaw University of Technology, Poland.
Received: December 30, 2019

Published: February 11, 2020

(C) All rights are reserved by Batyuk $L$ and

Kizilova N.

DOI: $10.31080 /$ ASCB.2020.04.0207

\begin{abstract}
Radiation protection ability of the ultra-disperse nanodiamonds (UDD) is studied based on the measurements of dielectric permittivity of red blood cells (RBC) affected by cancer. Wistar rats with Guerin's carcinoma were treated by X-ray 5.8 Gy. Some rats received UDD with food during 5 days prior to the X-ray. The groups with UDD, X-ray, and both UDD and X-ray treatments were compared to the control group. The complex dielectric permittivity of the RBC was measured by microwave dielectrometry. It was shown, tumor development leads to the increase in the dielectric permittivity and relaxation frequency. The irradiation promotes further growth of the parameters, while UDD uptake leads to insignificant changes in comparison to the control group. Therefore, UDD occur the radioprotective effect promoting repair, compensation and restoration of body tissues that is demonstrated by normalization of the dielectric parameters of RBC.

Keywords: Red Blood Cells; Nanodiamonds; Cancer; X-Ray; Dielectric Permittivity, Dielectric Relaxation
\end{abstract}

\section{Introduction}

Modern therapy of tumors is based on knowledge of the biological effect of ionizing radiation, the nature of tumor growth and the need for modern prevention of the cascade of adaptation reactions, in which all systems of the body participate, and primarily the blood system [1,2]. The established convenient model for studying tumor exposure and radiotherapy in vivo/in vitro are red blood cells (RBC) $[3,4]$, which, in addition to the main function of the oxygen carrier, carry out transport functions by sorption both low-molecular and high-molecular compounds at their surfaces, amino acids and lipids, performing a protective function, adsorbing toxins and antibodies. In addition to the convenient model of cells for research purposes, RBC can be used as natural containers, loaded with certain way of drugs, which allows prolonging the effect of drugs in therapeutic doses for radiation therapy [5,6]. Structural rearrangements in the erythrocyte membrane under the influence of malignant disease and radiation lead to quantitative and qualitative changes in the composition of erythrocyte membranes $[7,8]$. Currently, UDD like nanodiamonds with $\mathrm{d} \sim 4$ - $8 \mathrm{~nm}$ are used in biological research, and for medical diagnosis and treatment [9].

It was established the introduction of UDD increases the induction period before the onset of the tumor node and prolongs the life of tumor-bearing animals. Anomalously high adsorption capacity, large specific surface area, abundance of free electrons on the surface (multiple radical-donor), a large number of oxygencontaining functional groups on the surface of crystals, chemical inertness of grain UDD makes it possible to use them as a possible therapeutic facilities in oncology $[10,11]$.
In this work the dielectric permittivity of the RBC membranes has been studied on the experimental model of Guerin's carcinoma.

\section{Materials and Methods}

Modern therapy of tumors is based on knowledge of the biological effect of ionizing radiation, the nature of tumor growth and the need for modern prevention of the cascade of adaptation reactions, in which all systems of the body participate, and primarily the blood system [1,2]. The established convenient model for studying tumor exposure and radiotherapy in vivo/in vitro are red blood cells (RBC) [3,4], which, in addition to the main function of the oxygen carrier, carry out transport functions by sorption both low-molecular and high-molecular compounds at their surfaces, amino acids and lipids, performing a protective function, adsorbing toxins and antibodies. In addition to the convenient model of cells for research purposes, RBC can be used as natural containers, loaded with certain way of drugs, which allows prolonging the effect of drugs in therapeutic doses for radiation therapy $[5,6]$. Structural rearrangements in the erythrocyte membrane under the influence of malignant disease and radiation lead to quantitative and qualitative changes in the composition of erythrocyte membranes $[7,8]$. Currently, UDD like nanodiamonds with $\mathrm{d} \sim 4-8 \mathrm{~nm}$ are used in biological research, and for medical diagnosis and treatment [9].

It was established the introduction of UDD increases the induction period before the onset of the tumor node and prolongs the life of tumor-bearing animals. Anomalously high adsorption capacity, large specific surface area, abundance of free electrons on the surface (multiple radical-donor), a large number of oxygen-containing functional groups on the surface of crystals, chemical inertness of 
grain UDD makes it possible to use them as a possible therapeutic facilities in oncology $[10,11]$.

In this work the dielectric permittivity of the RBC membranes has been studied on the experimental model of Guerin's carcinoma.

\section{Materials and Methods}

Forty mature Wistar rats, weighing 160 - $180 \mathrm{~g}$ with subcutaneously transferred $20 \%$ suspension of Guerin's carcinoma cells were used for the studies. Rats were kept in vivarium on a standard diet fully accredited by the Association for Assessment and Accreditation of Laboratory Animal Care (AAALAC) [12]. The experimental group with Guerin's carcinoma of 20 rats received 1.0 $\mathrm{ml}$ of a diluted suspension of UDD once per day with food during 5 days prior to the radiation treatment. The suspension has $\mathrm{C}=$ $0.01 \%$ of dry weight of UDD in saline. The control group of 20 rats has not received UDD with food. Therefore, four groups of 10 rats have been studied: with both UDD and X-ray (I); with UDD without X-ray (II); without UDD with X-ray (III); with neither UDD nor Xray (IV). In that way, 20 rats from the groups I and III were treated by X-ray with a dose of $5.8 \mathrm{~Gy}$. Biological control included 10 rats. Blood samples were collected in 25 days after the irradiation.

The irradiation of rats was carried out in the device RUM-3M, the voltage on the tube of $190 \mathrm{kV}$, the current of $12 \mathrm{~mA}$, filters 0,5 $\mathrm{Cu}+1,0 \mathrm{Al}$, tubes $-40 \mathrm{~cm}$, the dose rate of $0,52 \mathrm{~Gy} / \mathrm{min}$, doses of 5.8 Gy [6].

The study of changes in dielectric properties of RBC has been carried out by the microwave dielectrometry at a frequency $\mathrm{f}=$ $9.2 \mathrm{GHz}$, based on the relative measurements of the real $\left(\varepsilon^{\prime}\right)$ and imaginary $\left(\varepsilon^{\prime \prime}\right)$ parts of the complex permittivity $\varepsilon^{*}$, and the static dielectric permittivity $\varepsilon_{s}$, the dielectric relaxation frequency of water molecules and electric conductivity have been then computed according to [13].

\section{Results and Discussion}

The temperature dependences of $\sigma, \varepsilon^{\prime}$ and $f_{d}$ in the RBC suspensions for different groups of rats are presented in Tables 1-3 and Figures 1 - 2. Table 1 shows that for the RBC suspension the temperature dependence of the specific conductivity of the RBC suspensions for different groups of animals is not linear and characterized by a step-like change of $\sigma$ values. In the rats with Guerin's tumor (group IV) the values of are bigger than those in the control group. The observed changes are associated with the presence of tumor toxins in the blood and changes in the viscosity and permeability of the erythrocyte plasma membranes in the presence of Guerin's carcinoma and, as a consequence, indicate a change in the amount of the free/bound water ratio in the erythrocytes membranes. The change in the free/bound water ratio is an integral feature that reflects, among other things, the characteristics of lipid and carbohydrate metabolism, since membrane fluidity (the value inverse to viscosity) depends on the membrane structure and interactions of protein-lipid and lipid-lipid complexes in it.

\begin{tabular}{|l|c|c|}
\hline $\mathbf{T}^{\circ} \mathbf{C}$ & Control group $\sigma, \mathrm{Ohm}^{-1} \cdot \mathrm{m}^{-1}$ & Group IV $\sigma, \mathrm{Ohm}^{-1} \cdot \mathrm{m}^{-1}$ \\
\hline 5 & $0.88 \pm 0.06$ & $1.22 \pm 0.09^{*}$ \\
\hline 10 & $1.02 \pm 0.01$ & $1.30 \pm 0.03^{*}$ \\
\hline 20 & $1.147 \pm 0.03$ & $1.47 \pm 0.04^{*}$ \\
\hline 30 & $1.17 \pm 0.03$ & $1.49 \pm 0.03^{*}$ \\
\hline 40 & $1.27 \pm 0.04$ & $1.55 \pm 0.01^{*}$ \\
\hline
\end{tabular}

Table 1: The temperature dependence of values of the electrical conductivity of samples of RBC suspensions for rats of group of biological control and rats of group IV.

Note: ${ }^{*}$ - reliability of differences in comparison with the biological control, $\mathrm{p} \leq 0.05$.

Comparison of the electric conductivity of RBC suspensions from table 2 in Group II with Group III and Group IV shows a tendency to decrease in this index over the entire temperature range. This trend is most pronounced for group II, that finds confirmation in the changes in the dielectric permittivity index (Figure $1 \mathrm{~A}-\mathrm{C}$ ) and the index of the dielectric relaxation frequency of water molecules in the erythrocyte suspension (Figure 2 a-c). Probably, destruction of the boundary layers of water in the erythrocyte membranes is observed, and the destruction is even amplified by the temperature growth that are accompanied by an increase in the electrophoretic mobility of UDD. UDD are a cation exchange system with a wide range of functional groups, forming an ion-permeable structure. The observed nature of changes is probably connected with the presence near the UDD surface thin water layers with a changed structure, and with the immobilization of the dispersion medium inside the UDD, which contributes to the change in the free/bound water ratio. in the RBC. The water immobilized inside the secondary aggregate of UDD may not participate in the movement, and the mobility of the ion exchange is preserved.

\begin{tabular}{|l|c|c|}
\hline T, ${ }^{\circ} \mathrm{C}$ & Group II $\sigma, \mathrm{Ohm}^{-1} \cdot m^{-1}$ & Group III $\sigma, \mathrm{Ohm}^{-1} \cdot m^{-1}$ \\
\hline 5 & $0,98 \pm 0.03^{* *}$ & $1.12 \pm 0.03^{* *}$ \\
\hline 10 & $1,20 \pm 0.03^{* *}$ & $1.20 \pm 0.03^{* *}$ \\
\hline 20 & $1.31 \pm 0.04^{* *}$ & $1.36 \pm 0.05^{* *}$ \\
\hline 30 & $1.34 \pm 0.03^{* *}$ & $1.39 \pm 0.03^{* *}$ \\
\hline 40 & $1.4 \pm 0.08^{* *}$ & $1.45 \pm 0.04^{* *}$ \\
\hline
\end{tabular}

Table 2: The temperature dependence of values of the electrical conductivity of samples of RBC suspensions for rats of group IIand group III.

Note: ${ }^{* *}$ - reliability of differences in comparison with the Group IV, $\mathrm{p} \leq 0.05$

On the other hand, for Group III the ionizing radiation initiates peroxidative chain reactions in unsaturated lipids and can upset the balance between peroxidizing agents $\left(\mathrm{O}_{2}, \mathrm{H}_{2} \mathrm{O}_{2}\right)$ and products of their reaction (hydroxyl radical HO- and singlet oxygen) and enzymatic protective systems (catalase and glutathione peroxidase, superoxide dismutase). This process leads to a metabolic amplifi- 
cation of the initial physicochemical radiation damage. As it was shown in, the whole-body -irradiation of rats (8 Gy) produced increased lipid peroxidation in several tissues and body fluids, including erythrocytes and blood plasma. Therefore all effects connected with the exposure dose could be related with oxidative stress of cells and result in more severe damage to lipids and proteins, especially sulphydryl (SH) groups in RBC membranes.

In the group I (Table 3), this indicator is levelled up to the biological control indicator. UDD exhibited radioprotection properties in respect of RBS membranes.

\begin{tabular}{|l|c|c|}
\hline $\mathbf{T},{ }^{\circ} \mathrm{C}$ & Control group $\sigma, \mathrm{Ohm}^{-1} \cdot \mathrm{m}^{-1}$ & Group I $\sigma, \mathrm{Ohm}^{-1} \cdot \mathrm{m}^{-1}$ \\
\hline 5 & $0.88 \pm 0.06$ & $0.82 \pm 0.03^{*}$ \\
\hline 10 & $1.02 \pm 0.01$ & $1.05 \pm 0.03^{*}$ \\
\hline 20 & $1.147 \pm 0.03$ & $1.145 \pm 0.08^{*}$ \\
\hline 30 & $1.17 \pm 0.03$ & $1.2 \pm 0.05^{*}$ \\
\hline 40 & $1.27 \pm 0.04$ & $1.306 \pm 0.05^{*}$ \\
\hline
\end{tabular}

Table 3: The temperature dependence of values of the electrical conductivity of samples of RBC suspensions for rats of group of biological control and rats of group I.

Note: ${ }^{*}$ - reliability of differences in comparison with the biological control, $\mathrm{p} \leq 0.05$.

The high oxygen content in UDD and the number of possible functional oxygen-containing groups on the surface of particles of nano scale sizes may lead to the fact that X-ray radiation could generate secondary electrons in UDD which can ionize water molecules to produce reactive oxygen species radical, resulting in DNA damage in cancer cells and lead to stabilization of RBC membranes. Possible, UDD caused more cancer cells damage in the presence of $\mathrm{X}$-ray radiation by generating more reactive oxygen species. UDD had long blood circulation time that enhanced their accumulation in cells.

The temperature dependence of dielectric permittivity and dielectric frequency of water molecules of samples of RBC suspensions for groups of animals with Guerin carcinoma, X-ray exposure and UDD Increased dielectric permittivity of the RBC suspension in the group IV that has been revealed by our study can be explained by a change in the absorption capacity of the RBC membranes involved in the neutralization of different endogenous toxins of tumours in blood. The given fact indicates the toxic load increase and the correspondent increase in the extent of the RBC membrane damage during tumour progression and toxins production. The sorption capacity of the RBC increases on the background of the growth malignant tumours which leads to an increase in the electrical conductivity of the cell (Table 1). This indicates the increase in the levels of tumour toxins circulating in the blood of rats and correspondingly the increase of the RBC membrane damage. Increased sorption capacity of RBC may lead to saturation of the cell membranes with toxic products, later resulting in increases in toxic substances in the blood plasma as well.67 The effects of circulating toxins during the tumour development may lead to conformational changes in the membrane proteins and phospholipids, increase in the membrane viscosity, changes in the RBC membrane structure, and as a result formation of more rigid membranes.

As it can be seen from Figure 1 that the values of the RBC suspensions in group IV and group III are bigger in comparison with the same values in the control group. The nature of changes in the values of RBC suspensions in the groups under study is most likely due to the non-monotonicity in the dependence of the amount of water bound by the cells influenced by the tumours, UDD, and $\mathrm{X}$-ray. However, since the values of the frequency of the dielectric relaxation of water molecules in the cell suspensions increase (Figure 2a), one can speak about an increase in the amount of free (bulk) water in the sample. At the present time, a lot of facts have accumulated, indicating the destructive effect of the ionizing radiation on various functional systems of the organism. At the same time, subtle mechanisms of pathological changes occurring at the cellular level require additional studies. The biological membranes of erythrocytes are assigned the role of a critical superstructure, any of its main functions being vital for the cell, and the damage to these functions can lead the cell to death. It is known that the biological feature of the action of the ionizing radiation is manifested, first of all, in the property of a "total stimulus". It is believed that at a dose of 5.8 Gy the leading role in determining the radiation responses of the cells is played not by irreversible damage to the genetic apparatus, but by reversible violations of the membrane structures that support the vital activity of the cell and its regulation. It is known that the physical processes underlying radiation damage to membranes can be either a direct effect of radiation, which leads to the absorption of the energy of ionizing radiation by membrane components or the effect of an indirect action associated with damage to protein and lipid cell molecules by free radical radiolysis products of the medium [13]. As is known, the permeability of biological membranes lays in the ability of various metabolites (amino acids, sugars, ions, etc.) to pass into/out the cell through the membrane. It is of great importance for osmoregulation and maintaining the constancy of the cell composition. Water is considered as an organizing factor, by means of which a spatial bilayer membrane structure is formed.

In the group I, after irradiating the animals received UDD with food, the values of the frequency of the dielectric relaxation of water molecules of RBC suspensions are significantly decreased in comparison with the control group (Figure 2C). Comparing the values in group I and in the control group, it can be assumed that radiotherapy, together with UDD, promotes the transition of water from the bound state to free, which leads to an increase in its mobility. The results observed in group I, when taking UDD before irradiation show approximation of the values of dielectric permittivity in the RBC suspensions to the values of dielectric permittivity in the control group (Figure 1C). This could be due to the fact that parallel to the processes of tissue damage under the influence of X-ray irradiation occur repair, compensation and restoration of body tissues after radiation damage, i.e. it becomes possible to implement protective adaptive reactions in the irradiated organ- 
ism, which is reflected in the normalized indices of permittivity of the RBC. The radioprotective effect of UDD at the cellular level, apparently, is not due to the inhibition of lipid peroxidation (LPO) in the membranes, but to the interception of $\mathrm{OH}$ - radicals formed during radiolysis of water. The involvement of LPO processes in the violation of the barrier properties of the RBC membranes under the influence of accelerated electrons seems unlikely for two reasons. First, the presence of a number of antioxidant systems is typical for erythrocytes, 75 which complicates the course of LPO reactions. Secondly, numerous studies have shown that the intensity of radiation-induced LPO is inversely proportional to the radiation dose rate. At the irradiation mode used in the present work, the average dose rate reached $0.52 \mathrm{~Gy} / \mathrm{min}$, so one should expect that the level of oxidative processes in the lipid phase of the membranes will be low. The radioprotective effect of UDD may be due to repair of lesions affecting SH- groups of proteins, or interception of free radicals arising from radiolysis of the medium. UDD having high chemically active fringe of functional groups on its surface (oxy, carboxy, carbonyl, etc.), increase the resistance of protein molecules to the effects of radiation, thus contributing to the restoration of radiation damage.
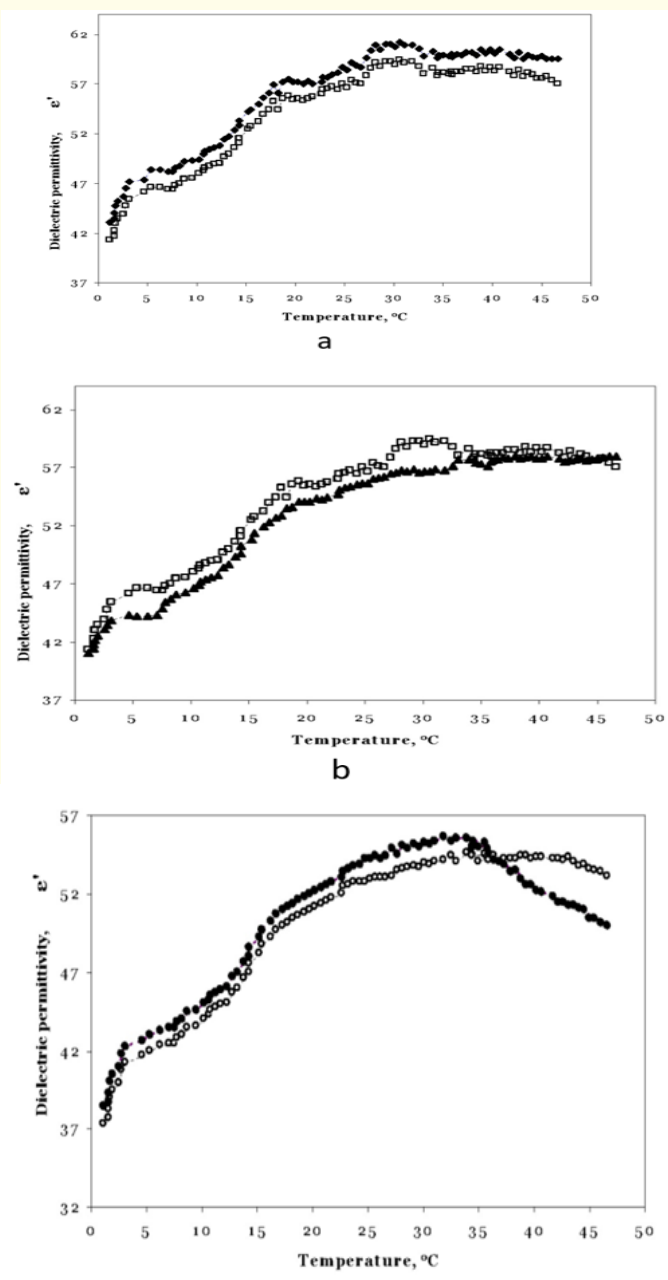

C

Figure 1: Temperature dependence of for the RBC suspensions of rats: $\notin$ - group I ; " - group II; $\square$ - group III; $\gamma$-group IV
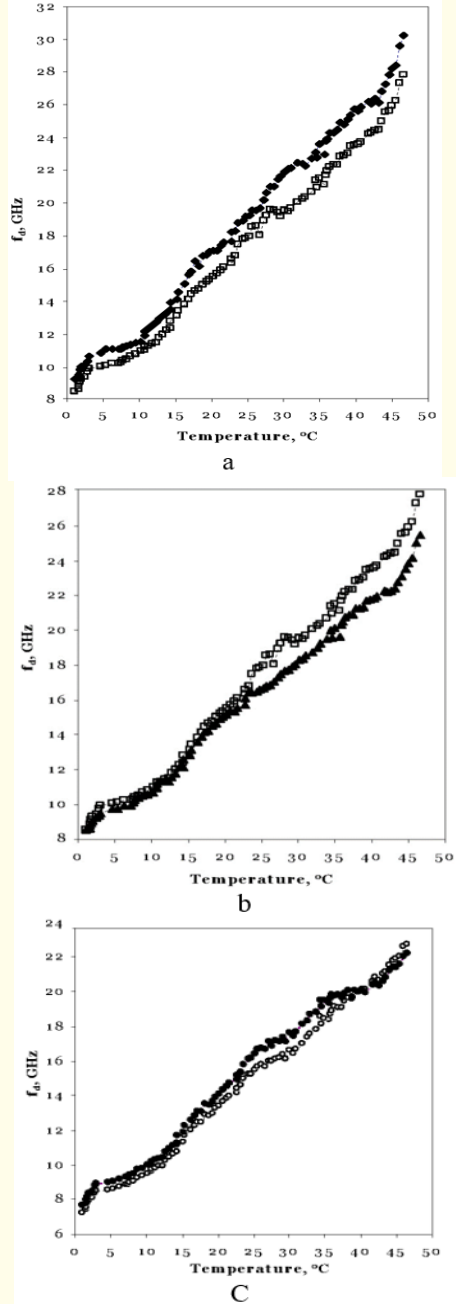

Figure 2: Experimental dependencies $\varepsilon^{\prime}(T)$ and $\varepsilon^{\prime \prime}(T)$ for the RBC suspensions of healthy donors $(\mathbf{\square})$, patients with breast cancer (") and lung cancer ( $\notin$ ).

\section{Conclusions}

Based on the dielectrometry measurement data on the temperature dependencies of The indirect radiation influence attack on the cell membranes may induce the membrane failure. Among the membrane disturbances are increase in rigidity (viscosity) of the lipid bilayer and aggregation of the membrane proteins. Based on the experimental data presented, one can conclude that the effect of a small dose of ionizing radiation under the conditions of Guerin's carcinoma model caused nonmonotonic changes in the erythrocyte permittivity indices, which indirectly indicates deeper structural and functional changes in RBC membranes under X-ray irradiation of rats. The multidirectional nature of the changes in the real and imaginary parts of the complex dielectric permittivity of RBC is probably due to the influence of intermolecular interactions, namely, a destruction of the grid of hydrogen bonds created by water molecules or UDD particles, that may be a consequence of the X-ray exposure in the conditions of Guerin's carcinoma model.

\section{Conflict of Interest}

The authors declare any neither financial interest or conflict of interests. 


\section{Bibliography}

1. Hanahan D and Weinberg RA. "Hallmarks of cancer: the next generation". Cell 144 (2011): 646-674.

2. Shahid S. "Review of hematological indices of cancer patients receiving combined chemotherapy and radiotherapy or receiving radiotherapy alone". Critical Reviews in Oncology/Hematology 105 (2016): 145-155.

3. Batyuk LV. "Influence of local fractionated X-ray irradiation on the hydration and structural state of erythrocytes at experimental models of carcinoma". Bulletin of Experimental Biology and Medicine 4 (2004): 135-140.

4. Batyuk LV., et al. "The dielectric permittivity in human erythrocyte membranes under action of radiation and combined treatment of cancer patients". In: The Intertational Multidisciplinary Regional Biophysics Meeting, Zreče, Slovenia (2005): 126.

5. Luk BT., et al. "Interfacial interactions between natural RBC membranes and synthetic polymeric nanoparticles". Nanoscale 6 (2014): 2730-2737.

6. Batyuk LV. "Influence of cancer disease on dielectric characteristic of structural-functional state of erythrocytes membranes". Medical Science Journal 7.4 (2015): 11-17.

7. Chitra S and Shyamaladevi CS. "Modulatory action of $\alpha$-tocopherol on erythrocyte membrane adenosine tri-phosphatase against radiation damage in oral cancer". The Journal of Membrane Biology 240 (2011): 83-88.

8. El-Say KM. "Nanodiamond as a drug delivery system: Applications and prospective". Journal of Applied Pharmaceutical Science 1 (2011): 29-39.

9. Dolmatov VY and Kostrova LN. "Nanodiamonds of detonation synthesis and the possibility of creating a new generation of drugs". Journal of Superhard Materials 3 (2000): 82-85.

10. Tsai LW., et al. "Nanodiamonds for medical applications: interaction with blood In vitro and In vivo". International Journal of Molecular Sciences 17 (2016): 9072-9077.

11. Gettayacamin M and Retnam L. "AAALAC International Standards and Accreditation Process". Toxicological Research 33 (2017): 183-189.

12. Batyuk L and Kizilova N. "Dielectric properties of red blood cells for cancer diagnostics and treatment". AS Cancer Biology 2 (2018): 55-60.

13. Benderitter M., et al. "The cell membrane as a biosensor of oxidative stress induced by radiation exposure: A multiparameter investigation". Radiation Research 159 (2003): 471-483.

\section{Assets from publication with us}

- Prompt Acknowledgement after receiving the article

- Thorough Double blinded peer review

- Rapid Publication

- Issue of Publication Certificate

- High visibility of your Published work

Website: https://www.actascientific.com/

Submit Article: https://www.actascientific.com/submission.php Email us: editor@actascientific.com

Contact us: +919182824667 